\title{
Frequency of nosocomial influenza cases in a tertiary hospital during the 2012-13 to 2014-15 seasons
}

\author{
JL Mendoza García, JG Mora Muriel, I Tenza Iglesias, P García Shimizu, M Fuster Perez, V García Roman, \\ JL Carretero Ares*, M El Attabi, J Sánchez Payá
}

From 3rd International Conference on Prevention and Infection Control (ICPIC 2015)

Geneva, Switzerland. 16-19 June 2015

\section{Introduction}

Control of influenza in the hospital must be a priority within the programs to improve patient safety.

\section{Objectives}

The objective is to determine the frequency and characteristics of nosocomial influenza cases in a tertiary hospital in the last three seasons.

\section{Methods}

We included all the patients hospitalized with suspected influenza during the 2012-13 and 2013-14 complete seasons; for the 2014-15, patients were included until week 10-2015. Cases were patients with influenza-like illness who were microbiologically confirmed (nasopharyngeal swabs or PCR positive for influenza virus A/B). Study variables were: type of infection (nosocomial -the onset of symptoms was 72 hours or more after the admission of the patient- / community-acquired), type of case (serious / not serious), death (yes / no). To study the association between seasons, chi-square test was used.

\section{Results}

Among the confirmed influenza cases, the frequency of nosocomial cases was $14.2 \%(18 / 127)$ in the $2012-13$ season, $11.6 \%(26 / 224)$ in the $2013-14$ season and $14.1 \%$ $(32 / 226)$ in the $2014-15$ season; $p=0.672$. The frequency of nosocomial cases among severe cases was: $7.5 \%(3 / 40)$ in the $2012-13$ season, $6.1 \%(4 / 66)$ in the 2013-14 season and $8.9(4 / 45)$ in the 2014-15 season; $\mathrm{p}=0.904$. The frequency of exitus in nosocomial cases was: $40 \%(2 / 5)$ in the $2012-13$ season, $0 \%(0 / 11)$ in the
2013-14 season and $8.3 \%(1 / 12)$ in the 2014-15 season; $\mathrm{p}=0.05$.

\section{Conclusion}

This study highlights the impact of nosocomial transmission of influenza within a tertiary hospital. The annual influenza vaccination of health professionals, together with recommendations on respiratory hygiene and an appropriate degree of compliance with droplet recommendations in the flu cases are the main strategies for the prevention of nosocomial influenza and therefore for the improvement of patient safety.

\section{Disclosure of interest}

None declared.

Published: 16 June 2015

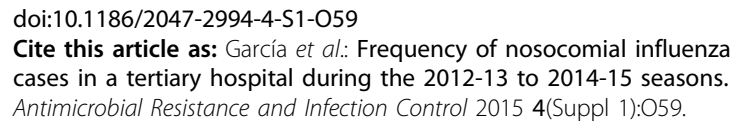

Hospital General Universitario de Alicante, Alicante, Spain 